\title{
A MULTI-TEMPORAL APPROACH FOR DETECTING SNOW COVER AREA USING GEOSTATIONARY IMAGERY DATA
}

\author{
Hwa-Seon Lee*, Kyu-Sung Lee \\ Dept. of Geoinformatic Engineering, Inha University, 100, Inha-ro, Nam-gu, Incheon, KOREA - hslee89@inha.edu
}

Commission VIII, WG VIII/6

KEY WORDS: snow cover, snow detection, multi-temporal, GOCI

\begin{abstract}
:
In this study, we attempt to detect snow cover area using multi-temporal geostationary satellite imagery based on the difference of spectral and temporal characteristics between snow and clouds. The snow detection method is based on sequential processing of simple thresholds on multi-temporal GOCI data. We initially applied a simple threshold of blue reflectance and then root mean square deviation (RMSD) threshold of near infrared (NIR) reflectance that were calculated from time-series GOCI data. Snow cover detected by the proposed method was compared with the MODIS snow products. The proposed snow detection method provided very similar results with the MODIS cloud products. Although the GOCI data do not have shortwave infrared (SWIR) band, which can spectrally separate snow cover from clouds, the high temporal resolution of the GOCI was effective for analysing the temporal variations between snow and clouds.
\end{abstract}

\section{INTRODUCTION}

Snow cover plays a key role in hydrology and climatology. In continental and global scale, the extent of snow cover area could be an indicator of climate change. In regional scale, snow melt is a major source of fresh water supply in certain regions, therefore snow water equivalent is important for the management of water resources. In local scale, snow cover monitoring is very critical for natural disaster preventions, such as flood and avalanche.

Conventional method for measuring snow cover area has been relied on point observations at ground weather stations. Since 1970s, satellite remote sensing has provided an opportunity to monitor snow cover area continuously. Optical sensor such as Moderate Resolution Imaging Spectroradiometer (MODIS) have been widely used to monitor snow cover area.

The snow detection algorithm used in MODIS snow product is mainly based on the spectral characteristic of snow in shortwave infrared (SWIR) wavelength. Because both snow and clouds have high reflectance in visible to near infrared (NIR) wavelengths, whereas snow has much lower reflectance than clouds in SWIR region due to absorption of solar radiation (Riggs et al., 2006). Therefore, SWIR band is essential to discriminate snow from clouds.

Although, MODIS snow product could provide useful information fully based on spectral characteristics of snow with high accuracy, it still contains some confusion errors between snow and clouds. While snow cover area has relatively constant surface condition over a certain time period, cloud covers are highly variable over short time period (Wildt et al., 2007). Combining both spectral and temporal characteristics of snow and cloud may enhance the separation of snow from clouds.

In this study, we attempt to detect snow cover area using multitemporal geostationary satellite imagery by both spectral and temporal characteristics of snow and clouds. The high temporal resolution images were obtained from the geostationary ocean color imager (GOCI) that provides eight hourly images during a day.

\section{DATA USED}

The GOCI is the first geostationary satellite sensor for monitoring ocean color at regional scale (Ryu et al., 2012). The GOCI provides eight hourly images per day with $500 \mathrm{~m}$ spatial resolution. Although, the main purpose of GOCI is to observe ocean color, GOCI also has great potential on land monitoring due to the high temporal resolution. It has eight spectral bands in visible and NIR wavelengths. Northern part of the GOCI target area has persistent snow cover in winter season. We used eight days GOCI L1B radiance images obtained from December 5 to 12 in 2011. To derive surface reflectance, atmospheric correction was applied using the $6 \mathrm{~S}$ radiative transfer model.

\section{SNOW DETECTION}

Initially, we analysed spectral characteristics of snow, clouds and other land cover types (vegetation and soil) that are observed in GOCI data. Both snow and clouds have high reflectance in GOCI spectral bands. Most land cover types have relatively low reflectance in blue wavelength. Thus simple threshold on blue reflectance was applied to separate snow and cloud pixels from other land covers.

Since GOCI does not have SWIR band, separation between snow and clouds was not easy due to the spectral similarity in visible and NIR wavelengths However, snow and clouds showed different temporal characteristics in reflectance. Although there was a certain diurnal variation in spectral reflectance because of the anisotropic behaviour, snow reflectance were almost constant pattern within certain temporal window (Figure 1). However, reflectance of clouds showed higher variation due to the high spatiotemporal variability (Figure 1).

To distinguish snow from clouds using multi-temporal observations, we used root mean square deviation (RMSD). The RMSD could represent the temporal deviation of spectral reflectance at specific time $t_{1}$ within a temporal window. 


$$
R M S D_{t_{1}}=\sqrt{\frac{\sum_{t_{1}}^{n} \sum_{t_{2}}^{n}\left(\rho_{t_{1}}-\rho_{t_{2}}\right)^{2}}{n-1}} \quad\left(\text { if } \mathrm{t}_{1} \neq \mathrm{t}_{2}\right)
$$

Where $\rho_{t_{1}}, \rho_{t_{2}}, n$ are surface reflectance at $t_{1}$ and $t_{2}$, and the number of observations within temporal window. If we use 3 days temporal window, then $\mathrm{n}$ is 24 (3days $\mathrm{x} 8$ observation per day). In most cases, RMSD of snow cover has lower than cloud cover. Shorter temporal window may include too little temporal information. On the other hand, longer temporal window will increase the temporal variability. Thus, the size of temporal window is important to determine the performance of snow detection.

The proposed snow detection method combines simple threshold on band reflectance and RMSD threshold calculated from multitemporal GOCI data. First step is to discriminate snow and cloud pixels using threshold of blue $(412 \mathrm{~nm}$, band 1$)$ reflectance. To avoid omission error, we applied relatively low threshold of 0.2 Second step is to classify snow cover and clouds using threshold of RMSD value of NIR reflectance $(865 \mathrm{~nm}$, band 8) calculated from multi-temporal GOCI data. This classification is only applied to those pixels of cloud and snow candidates that were extracted in the first step. We empirically selected 0.2 RMSD threshold of NIR band and 1day temporal window (eight observations).

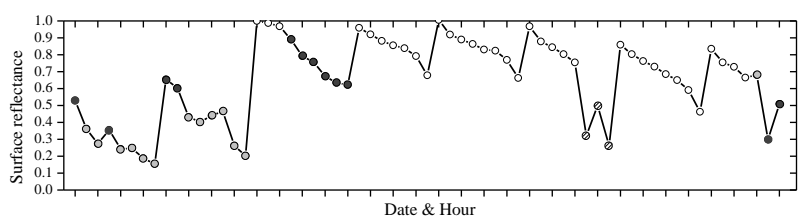

Figure 1. Temporal characteristics of snow and clouds on multitemporal GOCI data during eight days periods (white circle: clear, grey circle: clouds, stripe: shadow)

\section{RESULTS}

To validate the snow detection results obtained from the multitemporal GOCI data, we compared the snow detection results of proposed method to the MODIS daily snow product (MOD10A1). The daily MODIS snow product includes snow cover information, snow albedo, and snow fractional cover. Among them, snow cover information were used. To avoid time inconsistency, we used GOCI hourly image captured in 11:00 in local time, which is close to the image acquisition time of TerraMODIS.

The spatial distributions of snow cover detected from the proposed method using multi-temporal GOCI data were similar to the Terra-MODIS snow products (Figure 2-(b), Figure 3-(b)). However, snow cover on the northern part of GOCI image was somewhat different. The discrepancy between two outcomes can be verified by comparing with MODIS surface reflectance image (Figure 3-(a)). Snow cover and clouds can be well classified in MODIS SWIR color composite image by the strong absorption of snow in SWIR wavelength (orange: snow cover, white: clouds). MODIS snow products have some confusion errors despite of having SWIR spectral band. Snow cover and clouds could not be distinguished in GOCI color composite image because they have almost the same high reflectance (Figure 2(a)). Nevertheless, the proposed method showed more accurate snow detection results than MODIS snow product by adopting multi-temporal approach.

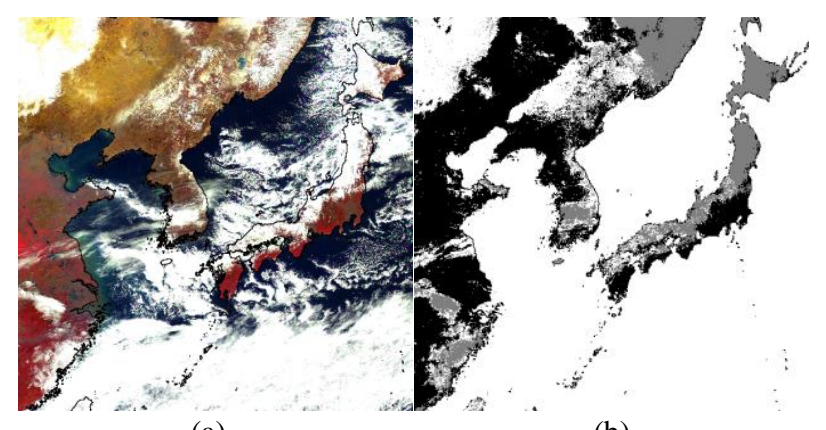

(a)

(b)

Figure 2. (a) NIR color composite GOCI surface reflectance image and (b) spatial distribution of snow cover detected by the proposed method from GOCI data in Dec 12, 2011 (white: snow cover, grey: clouds, black: clear land surface).

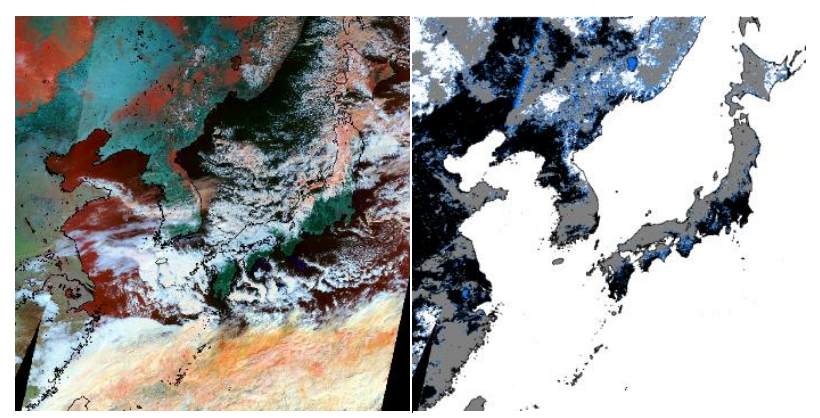

(c)

(d)

Figure 3. (a) SWIR color composite Terra-MODIS daily surface reflectance (b) Terra-MODIS daily snow products (MOD10A1) in Dec 12, 2011 (white: snow cover, grey: clouds, black: clear land surface, and blue: water and ice)

\section{CONCLUSIONS}

We developed a snow detection method using multi-temporal characteristics of snow reflectance on GOCI images. The proposed snow detection method uses both a simple threshold on band reflectance and RMSD threshold calculated from multitemporal GOCI data. The GOCI image has an inherent disadvantage of having only visible and NIR bands. Despite such limitations, the proposed method can detect snow cover with comparable accuracy. Combining both spectral and temporal characteristics of snow and clouds can enhance the capability of classifying snow cover areas.

\section{ACKNOWLEDGEMENTS}

This research was a part of the project titled 'Research for Applications of Geostationary Ocean Color Imager', funded by the Ministry of Oceans and Fisheries, Korea.

\section{REFERENCES}

[1] Riggs, G., D. Hall, and V. Salomonson, 2006. MODIS snow products user guide to collection 5, Digital Media: 80.

[2] de Wildt, Martijn de Ruyter, G. Seiz, and A. Gruen, 2007. Operational snow mapping using multitemporal Meteosat SEVIRI imagery, Remote Sensing of Environment, 109(1): $29-41$.

[3] Ryu, J., H. Han, S. Cho, Y. Park, and Y. Ahn, 2012. Overview of geostationary ocean color imager (GOCI) and GOCI data processing system (GDPS), Ocean Science Journal, 47(3): 223-233. 